\title{
Transmission autosomique du gène de la dystrophine chez le poulet
}

1. Lemaire C, Heilig R, Mandel JL. The chicken dystrophin cDNA : striking conservation of the C-terminal coding and 3' untranslated regions between man and chicken. EMBO $J 1988 ; 7: 4157-62$.

2. Dominguez-Steglich, Meng G, Betteken T, Müller CM, Schmid M. The dystrophin gene in autosomally located on a microchromosome in chicken. Genomics $1990 ; 8: 536-40$.

3. Sinclair AH, Foster JW, Spencer JA, et al. Sequences homologous to $\mathrm{ZFY}$, a candidate human sex-determining gene, are autosomal in marsupials. Nature $1988 ; 336: 780-3$.

4. Wilson B, et al. Major physiological and histochemical characteristics of inherited dystrophy of the chicken. Ann NY Acad Sa $1979 ; 317: 224-46$.

5. Hoffman P, Hudecki M, Pollina P, Rosenberg $P$, Kunkel L. Cell and fiber type distribution of dystrophin. Neuron $1988 ; 1$ : 411-20.
On sait que le gène de la dystrophine, protéine déficiente dans la myopathie de Duchenne, est porté par le chromosome X chez l'homme, comme chez la souris et le chien. L'ADNc correspondant à cette protéine a été cloné chez l'homme et la souris, et aussi chez le poulet. Il existe une homologie importante entre les ADNc du poulet et de l'homme [1]. Utilisant de l'ADNc humain, une équipe de Würzburg (RFA) a entrepris [2] de localiser le gène de la dystrophine du poulet, afin de vérifier si sa transmission s'effectue comme chez les mammifères par un chromosome sexuel. On sait que chez les oiseaux le sexe est régi par un système chromosomique $\mathrm{ZZ/ZW}$, dans lequel c'est la femelle qui constitue le sexe hétérogamétique : les oiseaux $\mathrm{ZZ}$ sont mâles, les ZW sont femelles. Il a été suggéré que l'X des mammiferes et le $\mathrm{Z}$ des oiseaux pourraient être homologues.

Les auteurs ont préparé des sondes couvrant les $14 \mathrm{~kb}$ de l'ADNc humain ; dans une première expérience un marquage radioactif de ces sondes suivi de Southern blots montra qu'il n'existait aucune différence quantitative entre mâles et femelles, rendant improbable une localisation du gène sur les chromosomes sexuels. Les mêmes sondes ont été ensuite marquées à la biotine (donc sans radioactivité) et soumises à une hybridation in situ avec des chromosomes en métaphase. Le marquage de chromosomes humains en Xp21 a d'abord été confirmé afin de valider la méthode; au contraire, chez le poulet, les chromosomes $Z$ n'étaient pas marqués, et la sonde était retrouvée hybridée à un des petits chromosomes autosomes (appelés microchromosomes), qui sont, dans l'espèce Gallus domesticus, au nombre de 30, à côté de 9 paires de macrochromosomes. On les classe par ordre de grandeur et le marquage est localisé sur les chromosomes 11, près du centromère. La localisation du gène de la dystrophine sur un autosome chez le poulet paraît ainsi solidement démontrée. Un tel phénomène n'est en fait pas si surprenant. Déjà chez des mammifères inférieurs (marsupiaux, monotrèmes), le gène de la dystrophine elle-même [3], ainsi que d'autres gènes homologues de ceux qui sont portés chez l'homme par le bras court de l'X (par exemple l'ornithine carbamyl transférase et la stéroïde sulfatase), sont autosomiques. Chez les oiseaux, la même démonstration a été apportée pour la glucose-6-phosphate déshydrogénase, la phospho-glycéro-kinase, l'hypoxanthine phosphoribosyltransférase. Réciproquement, les deux seuls gènes connus pour être localisés sur le chromosome $Z$, qui sont l'aconitase cytosolique du foie et la créatine kinase musculaire, sont autosomiques chez les mammifères [2]. Il apparaît donc impossible d'accepter l'hypothèse d'une origine ancestrale commune de l' $\mathrm{X}$ des mammifères et du $Z$ des oiseaux. Par ailleurs, ces observations pourraient redonner une actualité à la dystrophie musculaire du poulet, décrite en 1979 [4], dont on avait rejeté l'analogie possible avec la maladie humaine en raison de sa transmission autosomique. Elle ne semble toutefois pas liée à une forme mutée de dystrophine [5]. 